\title{
Cholesterol: An evergreen molecule in biology
}

\author{
G. Aditya Kumar and Amitabha Chattopadhyay* \\ CSIR-Centre for Cellular and Molecular Biology, Uppal Road, Hyderabad 500 007, India
}

\begin{abstract}
Cholesterol, an essential component of higher eukaryotic membranes, was discovered more than two centuries ago. The development and progress of cholesterol research in the last 200 years has been truly fascinating, with elements of surprise, serendipity and intrigue. In this review, we trace this journey the way we see it, and follow it up with the role of membrane cholesterol in crucial areas of contemporary research (transbilayer domains, regulation of GPCR function and role in the entry of intracellular pathogens into host cells), with considerable footprint from our work. We believe that cholesterol will continue to surprise and fascinate future researchers, thereby justifying its evergreen nature.
\end{abstract}

Keywords: Membrane cholesterol, transbilayer dimer, G protein-coupled receptor, pathogen entry

\author{
Abbreviations \\ 7-DHC \\ 7-DHCR \\ 25-NBD-cholesterol \\ CRAC \\ DHE \\ DPPC \\ FRAP \\ GPCR \\ NBD \\ REES \\ SLOS
}

\section{Cholesterol: An ancient molecule with a remarkable history}

Cholesterol was initially discovered as a component of human bile and gallstone sometime around 1760 AD by Poulletier de la Salle, a French physician and chemist (see Fig. 1) [13]. The naming of cholesterol was done by another French chemist Michel Chevreul in 1816. He named it cholesterine

\footnotetext{
${ }^{*}$ Corresponding author. E-mail: amit@ccmb.res.in.
} 


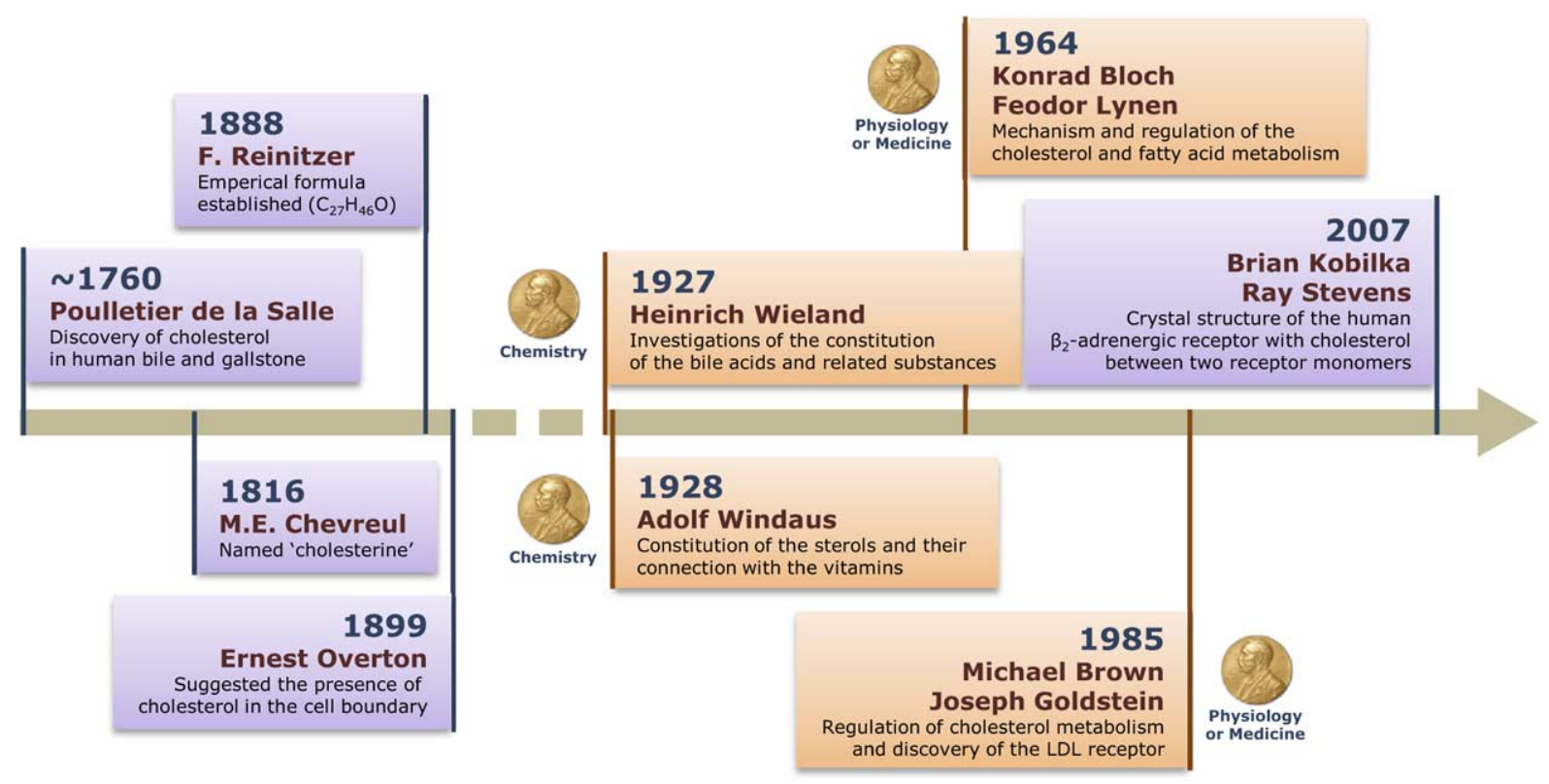

Fig. 1. The journey of cholesterol: 200 years and beyond. A timeline depicting landmark discoveries associated with cholesterol, and its structure, function and metabolism. Importantly, discoveries related to cholesterol have been recognized by the award of several Nobel Prizes, due to its importance in cellular physiology, health and disease.

(chole for bile and stereos for solid since it was discovered in human gallstone) [56]. The pace of scientific research in those times was slow by today's standards and it was only in 1888 that further characterization of cholesterol in terms of its empirical formula $\left(\mathrm{C}_{27} \mathrm{H}_{46} \mathrm{O}\right)$ was carried out by Friedrich Reinitzer, an Austrian botanist and chemist. The molecular structure of cholesterol was deduced by Henreich Weiland and Adolf Windaus, and both were awarded the Nobel Prize (although not in the same year) for their seminal work [56]. Paradoxically, the first structure proposed for cholesterol in 1928 was not correct! The correct structure of cholesterol was worked out by X-ray studies by J.D. Bernal and chemical studies by others $[2,56]$. The presence of cholesterol in cellular membranes was first suggested by Ernest Overton (generally known for the Meyer-Overton rule) toward the end of the nineteenth century [38]. A number of scientists (including the legendary chemist Robert B. Woodward) contributed to the molecular understanding of cholesterol biosynthesis. The complete biosynthetic pathway of cholesterol was worked out in the mid-twentieth century by Konrad Bloch [4]. Independently, Feodor Lynen from Germany also worked on the biosynthesis of cholesterol during the same time. Bloch and Lynen shared the Nobel Prize in Physiology or Medicine in 1964. Another important conceptual advance in cholesterol research came from the work of Michael Brown and Joseph Goldstein who discovered the low density lipoprotein (LDL) receptor which helped understand the regulation of cholesterol biosynthesis and metabolism in humans [6]. Their seminal discovery, relevant in a number of human diseases, was recognized by the award of the Nobel Prize in Physiology or Medicine in 1985.

In this review, we will first highlight some unique structural features of cholesterol that are closely related to its functionality. This will be followed up with highlights of the role of cholesterol in three representative areas of membrane biology. These topics (transbilayer domains, regulation of G proteincoupled receptor (GPCR) function and role in the entry of intracellular pathogens into host cells) are chosen primarily because of our previous and ongoing work in these areas. 


\section{Molecular idiosyncrasies of cholesterol: Key to functionality}

The molecular structure of cholesterol (see Fig. 2) is characterized by a number of distinct features, all of which combine to make cholesterol a unique molecule in functional terms. Cholesterol is a predominantly hydrophobic molecule comprising a near planar tetracyclic fused steroid ring and a flexible isooctyl hydrocarbon tail. The $3 \beta$-hydroxyl group provides cholesterol its much needed amphiphilic character and helps cholesterol to orient and anchor in the membrane. The tetracyclic nucleus and isooctyl side chain make up the rest of the molecule. The sterol ring has a flat and smooth side with no substituents (the $\alpha$ face) and a rough side with methyl substitutions (the $\beta$ face; see Fig. 2(b)) [11]. The two faces differ in their preferences for near neighbors. The smooth $\alpha$ face of the sterol nucleus, containing only axial hydrogen atoms, helps in energetically favorable van der Waals interaction with the saturated fatty acyl chains of phospholipids. The bumpiness of the rough $\beta$ face of cholesterol is due to the protruding methyl groups. Due to its bumpy nature, this face preferentially interacts with membrane proteins characterized by undulated topology.

The molecular structure of cholesterol is exceedingly fine-tuned over a very long time scale of natural Darwinian evolution. This is exemplified by a number of inherited disorders, such as the SmithLemli-Opitz syndrome (SLOS; see Fig. 2(c)), the molecular etiology of which lies in subtle defects in cholesterol biosynthesis $[3,35,40,42]$. SLOS is a congenital and developmental malformation syndrome associated with defective cholesterol biosynthesis. In SLOS, the immediate precursor in the KandutschRussel pathway of cholesterol biosynthesis is 7-dehydrocholesterol (7-DHC), which differs with cholesterol merely in a double bond, and is accumulated due to mutations in 7-DHCR. SLOS is clinically characterized by reduced levels of plasma cholesterol coupled with elevated plasma concentrations of 7-DHC.

\section{A unique transbilayer organization of membrane cholesterol at low concentrations: Physiological implications}

Cholesterol is an essential lipid in higher eukaryotic cellular membranes and is crucial in membrane organization, dynamics, function, and sorting [27,54]. A hallmark of the organization of membrane cholesterol is its nonrandom distribution in domains [30]. A unique property of cholesterol that contributes to its capacity to form membrane domains, when present at high concentrations, is its ability to form liquid-ordered-like phase in higher eukaryotic plasma membranes [26]. Many of these domains (sometimes termed as 'lipid rafts' [22]) are thought to be important for the maintenance of membrane structure and function, although characterizing the spatiotemporal resolution of these domains has proven to be challenging [53].

Interestingly, cholesterol is distributed heterogeneously among various intracellular membranes. The lowest cholesterol concentration is found in membranes of the endoplasmic reticulum, which is the site of cholesterol biosynthesis. Cholesterol concentration increases progressively along the cis, medial and trans Golgi stacks, while the highest concentration ( $\sim 90 \%$ of the total cellular cholesterol) is found in the plasma membrane [5]. Strangely, while a large body of literature exists on the organization of cholesterol in the plasma membrane (where cholesterol concentration is high, typically $>30 \mathrm{~mol} \%$ of total membrane lipids), very little is known about organization of cholesterol in membranes containing low amounts of cholesterol (such as the endoplasmic reticulum and mitochondrial membranes).

Evidence for specific organization of cholesterol in membranes at low concentrations was obtained from three independent studies carried out by various groups $([7,15,16,28]$; reviewed in [10]). Harris 


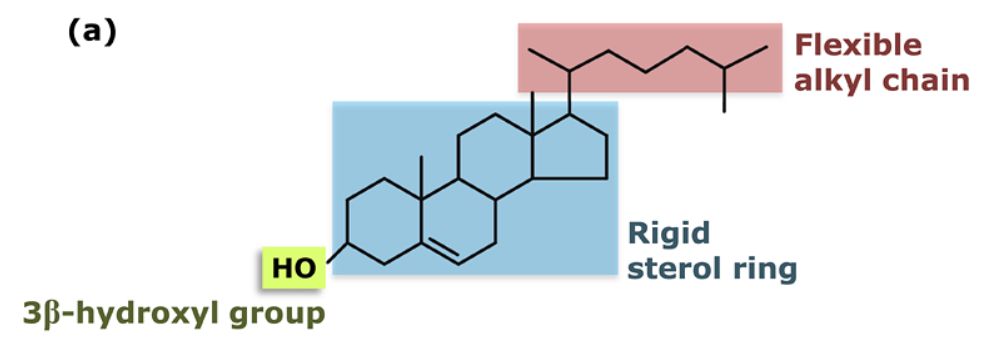

\section{(b)}

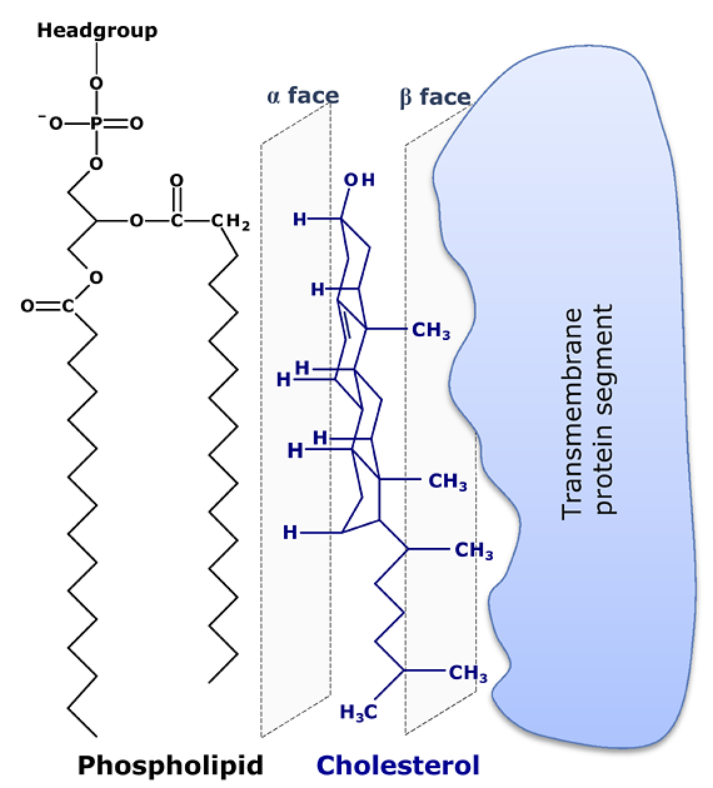

(c)

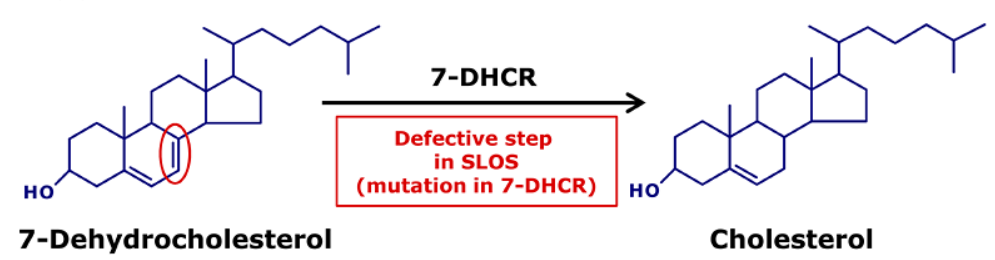

Fig. 2. Key structural features of cholesterol and its interaction with membrane proteins and diseases resulting from defective cholesterol biosynthesis. (a) Chemical structure of cholesterol depicting its three structurally distinct regions, each outlined in a shaded box: a polar $3 \beta$-hydroxyl group (green), a rigid near-planar tetracyclic sterol ring (cyan), and a flexible acyl chain (red). The chemical features of each of these regions are stringently controlled and fine-tuned by various steps in biosynthesis. The combination of polar ( $3 \beta$-hydroxyl group) and apolar (the sterol ring and the isooctyl side chain) regions impart an amphipathic nature to cholesterol, making it conducive to interaction with other membrane components (lipids and proteins). (b) A schematic representation of the orientation of cholesterol in membranes with respect to phospholipids and membrane proteins. Cholesterol aligns in the membrane bilayer such that its polar hydroxyl group interacts with the ester carbonyls of phospholipids and its apolar part orients along the phospholipid fatty acyl chain. An interesting structural feature of cholesterol is the inherent asymmetry about the sterol ring plane owing to methyl substitutions on one of its faces. The smooth $(\alpha)$ face of cholesterol constituting of only axial hydrogen atoms contributes to favorable van der Waals interaction with the saturated fatty acyl chains of phospholipids. On the other hand, the rough $(\beta)$ face characterized by the protruding methyl groups can snugly interact with the bumpy topology of a membrane protein. Adapted and modified from [10]. (c) Molecular etiology of SLOS: defective cholesterol biosynthesis pathway leads to accumulation of 7-DHC, an immediate biosynthetic precursor of cholesterol, differing with cholesterol merely in a double bond (highlighted). The defect in the biosynthesis is caused by mutations in the enzyme 7-DHCR. 
et al. [16] monitored the cryoscopic depression of multilamellar vesicles of DPPC/cholesterol and explained their results on the basis of transbilayer dimer formation in the fluid phase at low concentrations. Differential scanning calorimetric studies showed that there was a sharpening of the phase transition (due to increase in the size of cooperative unit) by cholesterol at very low concentrations, instead of the broadening expected if chain melting was accompanied by continuously changing compositions of the two phases [16]. Further analysis of cholesterol-DPPC interaction, monitored by lowering of transition temperature as a function of increasing cholesterol concentration in DPPC membranes showed that the slope of the curve (of transition temperature $v s$. cholesterol concentration) was consistent with the molecular weight of a dimer when cholesterol concentration was $3 \mathrm{~mol} \%$ and above. These results showed that while cholesterol is immiscible in the gel phase membrane and exists as separate solid domains, it forms monodisperse solutions below $2 \mathrm{~mol} \%$ in the fluid phase. Based on space-filling considerations and control experiments with sterols lacking the isooctyl chain, these authors proposed a transbilayer dimer arrangement stabilized by van der Waals interaction (see Fig. 3(a)). In this model, cholesterol molecules are localized rather deep in the membrane.

Mukherjee and Chattopadhyay [28] utilized aggregation-sensitive fluorescence characteristics of 25NBD-cholesterol, a fluorescent analogue of cholesterol, to study the local organization of cholesterol in the membrane at very low concentrations. Lipids labeled with the NBD group have been extensively used as fluorescent probes to monitor membrane environment and dynamics due to a number of excellent photophysical properties of the NBD group [7,15]. By careful analysis of the emission spectral features of 25-NBD-cholesterol in DPPC membranes at low concentration, the possible presence of transbilayer tail-to-tail dimers (Fig. 3(a)) of cholesterol in such membranes was detected both in gel and fluid phases.

These results were further validated by Loura and Prieto [23] by utilizing the intrinsic fluorescence of DHE, a naturally occurring fluorescent cholesterol analogue found in yeast. Loura and Prieto [23] analyzed DHE concentration-dependent depolarization in membranes using a theoretical framework and explained their results on the basis of transbilayer tail-to-tail dimers of cholesterol, previously reported by us [28] and others [16]. These conclusions were further supported by measurements in which the change in peak intensity ratio of the vibronic bands of DHE was monitored as a function of DHE concentration [50]. In addition, it was indicated that cholesterol dimers are sensitive to membrane curvature [50].

The effect of membrane curvature stress on the transbilayer organization of cholesterol dimers in membranes was comprehensively addressed by us [50]. By monitoring the distinct spectral feature of 25-NBD-cholesterol in membranes of varying curvature, we showed that the transbilayer dimer arrangement is sensitive to membrane curvature and dimerization is not favored in highly curved membranes (see Fig. 3(b)). This was attributed to differential packing arrangements in the two leaflets of highly curved membranes. Interestingly, cholesterol dimerization was favored upon release of curvature stress [50]. Furthermore, the transbilayer cholesterol dimers exhibited sensitivity to membrane thickness (see Fig. 3(a)). Taken together, these results showed that the process of transbilayer dimerization is stringently controlled by a narrow window of membrane thickness and curvature (see Fig. 3(a), (b)). These results assume relevance in the context of increasing membrane thickness from Golgi to the plasma membrane due to the gradient in cholesterol concentration [5]. We later showed, using wavelengthselective FRAP [46] and REES [29] that the dynamics of the cholesterol monomer and dimer population is considerably different. The monomer population exhibited faster lateral dynamics and was found to be less ordered than the dimer (Fig. 3(c)). 
(a)

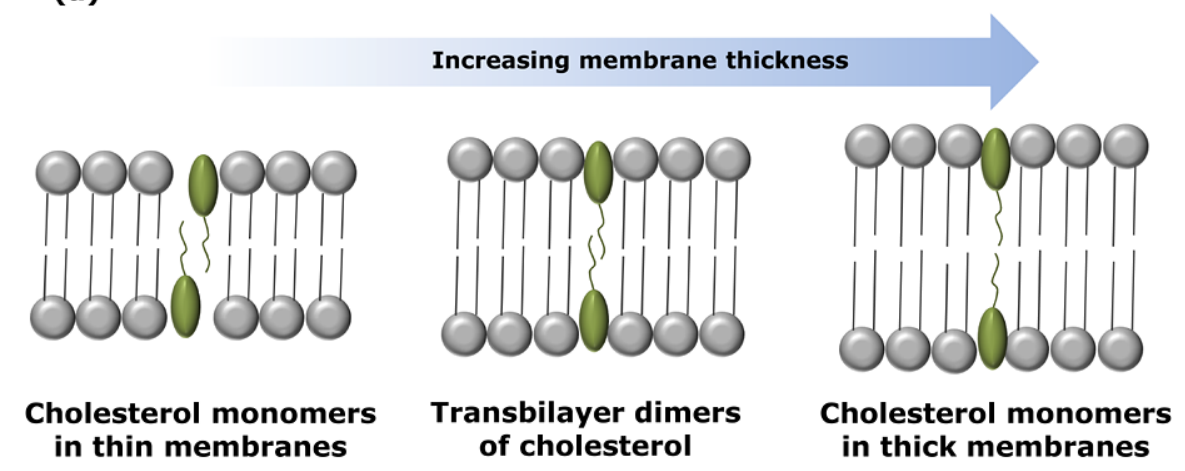

(b)

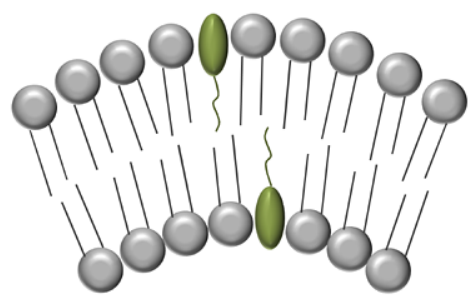

Cholesterol monomers in highly curved membranes

(c)

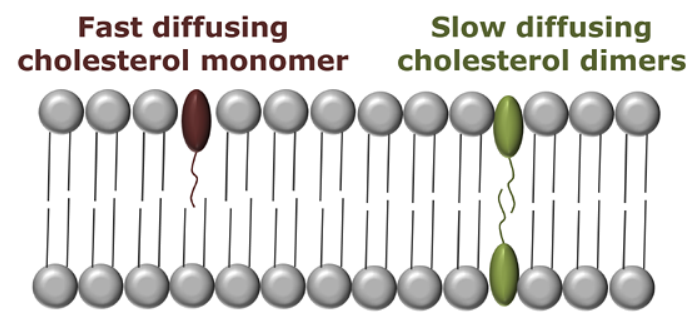

Fig. 3. Organization and dynamics of transbilayer dimers of cholesterol at low concentrations. The schematic diagram shows the organization of cholesterol dimers in (a) membranes of varying thickness. The existence of cholesterol as transmembrane tail-to-tail dimers is stringently controlled within a narrow window of membrane thickness. Insights from our previous work suggest that cholesterol exists as monomers in relatively thin and thick membranes, while it forms transmembrane tail-to-tail dimers in membranes of intermediate (optimum) thickness. (b) Cholesterol dimers are unstable at high surface curvature. (c) Monomers of cholesterol show faster lateral dynamics in membranes relative to transbilayer tail-to-tail dimers of cholesterol (see [46] for details).

\section{Role of membrane cholesterol in GPCR organization and function: What have we learnt?}

GPCRs are the largest class of molecules involved in signal transduction across membranes, and represent major targets in the development of novel drug candidates in all clinical areas [8,39,49]. Since GPCRs are integral membrane proteins, interaction of membrane lipids with them constitutes an important area of research in GPCR biology. In particular, membrane cholesterol has been reported to have a modulatory role in the function of a number of GPCRs [14,17,31,34,44,52]. Extensive work has been carried out in case of GPCRs such as the $\operatorname{serotonin}_{1 \mathrm{~A}}$ receptor, the $\beta_{2}$-adrenergic receptor, and 


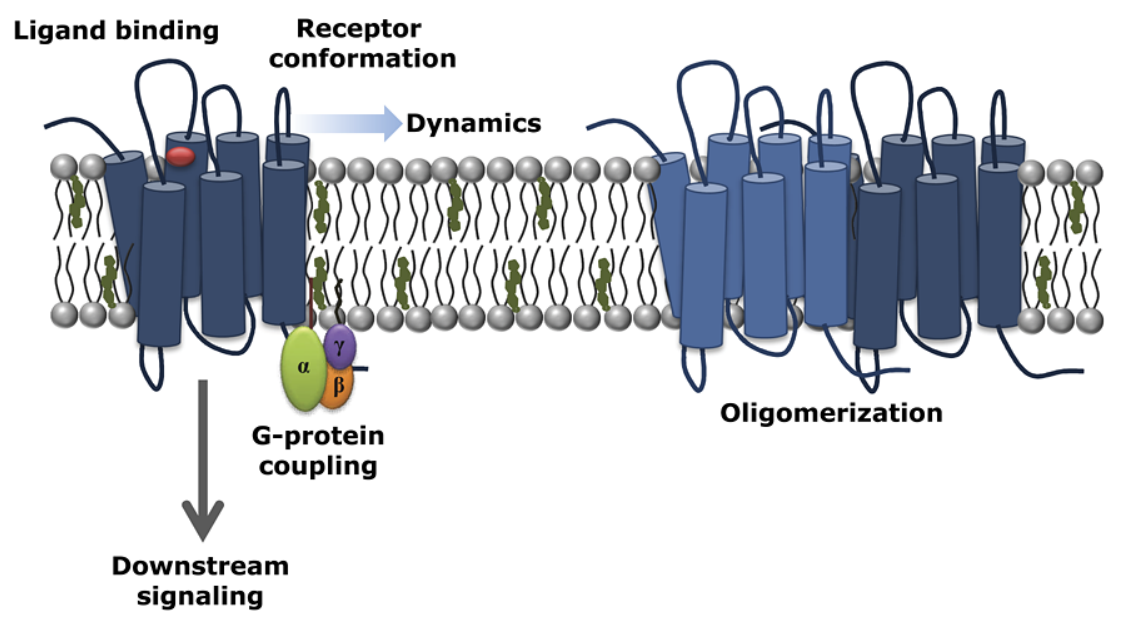

Fig. 4. The multifaceted interaction of cholesterol with GPCRs. A schematic representation highlighting the consequences of modulation of membrane cholesterol (shown in green) in GPCR structure, function and organization. Cholesterol has been shown to modulate early events associated with GPCR function such as ligand binding and G-protein coupling, leading to consequences in downstream signaling. Experimental and computational evidences show that membrane cholesterol modulates the organization, dynamics and oligomerization of GPCRs. Such an intimate interplay between GPCRs and cholesterol could arise either due to specific interaction of cholesterol with hotspots on GPCRs where cholesterol enjoys higher occupancy, or due to modulation of membrane physical properties, or a combination of these effects (see $[14,17,31,34,44,52]$ for more details).

the cannabinoid receptor. In all these cases, membrane cholesterol regulates GPCR function, dynamics and oligomerization (see Fig. 4). Details of these effects have been described in previous reviews $[14,17,31,34,44,52]$ and we direct interested readers to these reviews.

We will instead briefly focus on the current understanding of the mechanistic basis of GPCRcholesterol interaction. It appears that specific effects in terms of cholesterol binding to certain regions (sequences) of the receptor plays a role in these changes [33,37]. Some of these regions (such as the CRAC motif) have been identified [18]. The involvement of these regions in regulation of GPCRs by membrane cholesterol is being investigated for various types of GPCRs by experimental [32] and simulation approaches [51]. Detailed molecular dynamics simulations have revealed that cholesterol binding on GPCRs is weak yet dynamic with an occupancy time varying between ns and $\mu$ s (Fig. 5(a)). The emerging model regarding the energy landscape of cholesterol association with GPCRs is that it corresponds to a series of shallow minima interconnected by low energy barriers (see Fig. 5(b)). This translates to GPCR conformational plasticity induced by membrane cholesterol with varying functional attributes [43].

\section{Role of host membrane cholesterol in pathogen entry}

The cellular plasma membrane serves as a gateway for the entry of intracellular pathogens. An essential step for an intracellular pathogen to enter into a host cell therefore is to be able to cross the cell membrane. A clever strategy to regulate the entry of the intracellular pathogen (thereby reducing infection) is to change host membrane composition such that pathogen entry is reduced. An effective way to achieve this is to reduce (or block) host membrane cholesterol. This approach has been successfully tried for entry of pathogens such as Leishmania [9,20,21,36,45,47,48,55] and Mycobacterium [12,20,57]. An advantage of such therapeutic approach involving modulation of host membrane cholesterol provides 
(a)

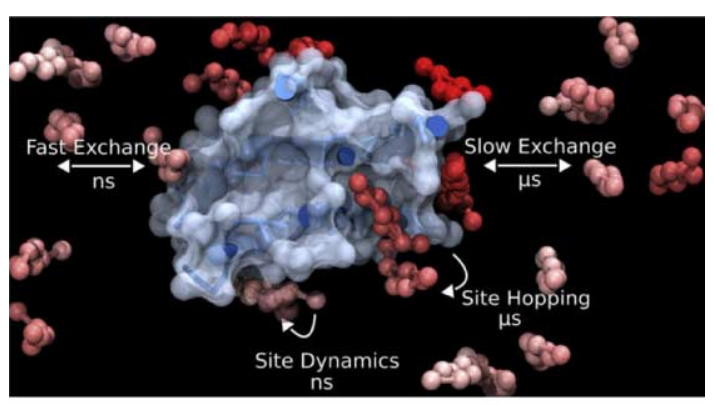

(b)

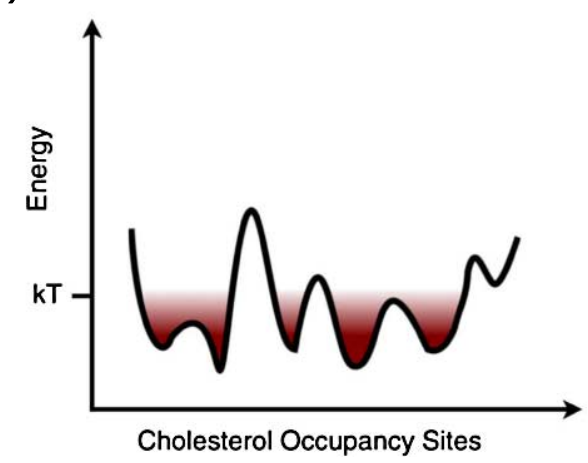

Fig. 5. Interaction of cholesterol with GPCRs: dynamic and diverse. (a) The interaction of cholesterol with GPCRs exhibits diversity in terms of its occupancy at the GPCR surface. The interaction is weak but crucial. Cholesterol molecules interacting with receptors exhibit varying timescales of exchange with bulk lipids. (b) The energy landscape corresponding to the interaction of cholesterol with specific sites on the receptor surface involves a series of shallow minima connected by low energy barriers of the order of $\mathrm{kT}$ at room temperature. Taken from ref. [52].

the advantage that the development of drug resistance, a common problem associated with treatment of diseases caused by these pathogens [1,19], would be absent since the therapeutic focus is on the host membrane cholesterol, rather than the pathogen.

We have recently proposed a working mechanism for this approach (see Fig. 6). The crux of the mechanism is that the conformation(s) of the host membrane receptors, responsible for pathogen entry, display cholesterol sensitivity. An optimum level of host membrane cholesterol is found to be necessary for supporting receptor conformation(s) that allow pathogen entry. Either depletion or enrichment of membrane cholesterol results in receptor conformation(s) that do not support the entry of pathogens into host cells (for details, see ref. [20]).

\section{Conclusion and the road ahead}

The examples discussed above represent chosen areas where cholesterol has a unique role in membrane organization, function and pathogenicity. The interesting and fascinating aspect of cholesterol in biology is that we are yet to comprehensively understand the whole gamut of biological roles it could play and the crosstalk between pathways influenced by cholesterol. Every once in a while, reports appear in the literature that make us re-think our existing models about cholesterol in biology. For example, 
(a)

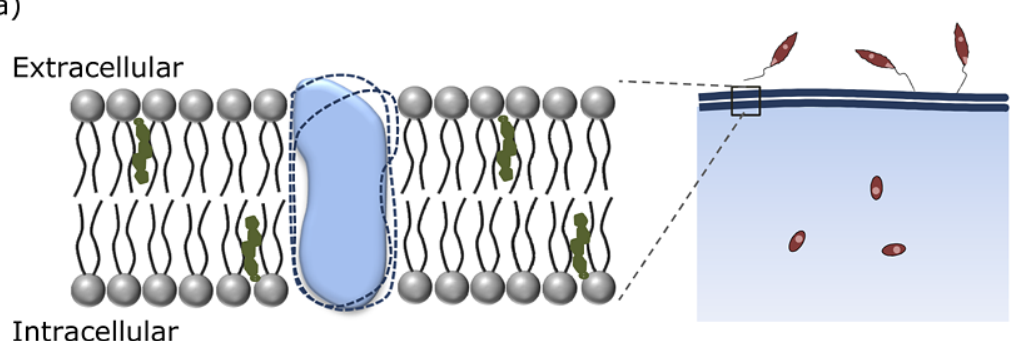

Intracellular

Depletion of membrane cholesterol

does not support pathogen entry

(b)

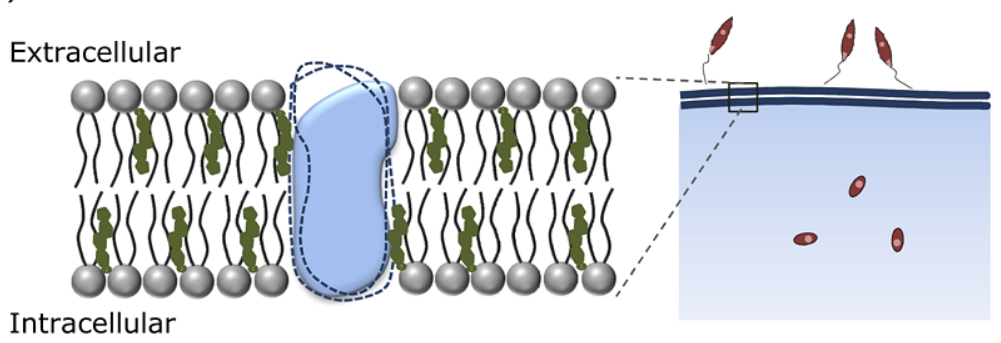

\section{Enrichment of membrane cholesterol does not support pathogen entry}

(c)

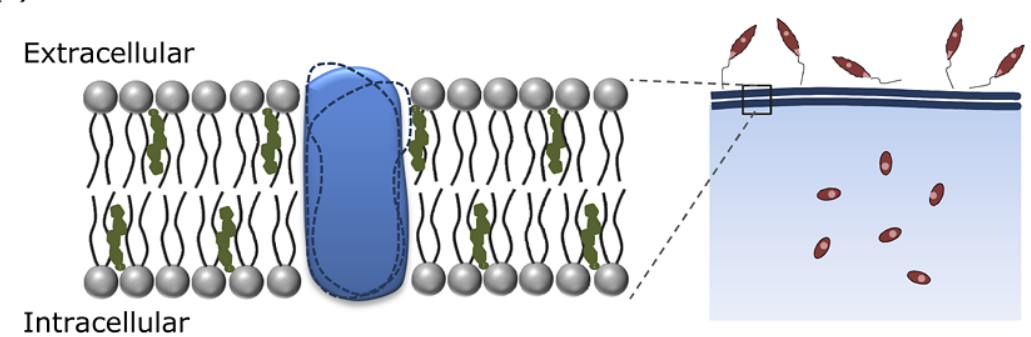

Optimum membrane cholesterol supports pathogen entry

Fig. 6. Regulation of pathogen entry by host membrane cholesterol. Host membrane cholesterol modulates the entry of intracellular pathogens (such as Leishmania, as shown in the figure). Previous results from our laboratory have shown that (a) depletion as well as (b) enrichment of membrane cholesterol in host cells results in reduced pathogen entry, while (c) an optimum level of host membrane cholesterol is required for efficient pathogen entry. We propose that such a cholesterol-mediated entry of intracellular pathogens could be due to the interaction of membrane cholesterol with host cell surface receptors implicated in pathogen entry. Receptor conformations stabilized in the membranes of varying cholesterol content are rendered in solid shapes, while dotted outlines depict other unfavorable conformations. Optimum cholesterol levels in host cells could support receptor conformation(s) that enable efficient pathogen entry. On the other hand, depletion or enrichment of host membrane cholesterol could render receptor conformation(s) that do not support the entry of intracellular pathogens.

cholesterol has been implicated in maturation and signaling of hedgehog proteins involved in embryonic development in Drosophila [41], and programmed cell death [24]. Another report appeared a few years back in which cholesterol was implicated, for the first time, in synaptogenesis in the central nervous system [25]. These examples justify the 'evergreen' nature of cholesterol, as alluded to in the title of our review. In summary, cholesterol has enriched biology in its two centuries of glorious existence. What remains to be seen is what new scenario evolves in relation to cholesterol in decades to come. Cholesterol, 
no doubt, will keep researchers engaged in this pursuit.

\section{Acknowledgements}

Work in A.C.'s laboratory was supported by the Council of Scientific and Industrial Research, Department of Science and Technology, and Department of Biotechnology (Govt. of India). A.C. gratefully acknowledges the support of J.C. Bose Fellowship from the Department of Science and Technology. G.A.K. thanks the Council of Scientific and Industrial Research for the award of a Senior Research Fellowship. A.C. is an Adjunct Professor of Tata Institute of Fundamental Research (Mumbai), RMIT University (Melbourne, Australia), and Indian Institute of Technology (Kanpur). Some of the work described in this article was carried out by present and former members of A.C.'s research group whose contributions are gratefully acknowledged. We thank Md. Jafurulla for help during the preparation of the manuscript and members of the Chattopadhyay laboratory for their comments and discussions.

\section{References}

[1] J. Berman, Current treatment approaches to leishmaniasis, Curr. Opin. Infect. Dis. 16 (2003), 397-401. doi:10.1097/ 00001432-200310000-00005.

[2] J.D. Bernal, Crystal structures of vitamin D and related compounds, Nature 129 (1932), 277-278. doi:10.1038/129277a0.

[3] S.E. Bianconi, J.L. Cross, C.A. Wassif and F.D. Porter, Pathogenesis, epidemiology, diagnosis and clinical aspects of Smith-Lemli-Opitz syndrome, Expert Opin. Orphan Drugs 3 (2015), 267-280. doi:10.1517/21678707.2015.1014472.

[4] K. Bloch, The biological synthesis of cholesterol, Science 150 (1965), 19-28. doi:10.1126/science.150.3692.19.

[5] M.S. Bretscher and S. Munro, Cholesterol and the Golgi apparatus, Science 261 (1993), 1280-1281. doi:10.1126/science. 8362242.

[6] M.S. Brown and J.L. Goldstein, How LDL receptors influence cholesterol and atherosclerosis, Sci. Am. 251 (1984), 58-66. doi:10.1038/scientificamerican1184-58.

[7] A. Chattopadhyay, Chemistry and biology of $N$-(7-nitrobenz-2-oxa-1,3-diazol-4-yl)-labeled lipids: Fluorescent probes of biological and model membranes, Chem. Phys. Lipids 53 (1990), 1-15. doi:10.1016/0009-3084(90)90128-E.

[8] A. Chattopadhyay, GPCRs: Lipid-dependent membrane receptors that act as drug targets, Adv. Biol. 2014 (2014), 143023.

[9] A. Chattopadhyay and M. Jafurulla, Role of membrane cholesterol in leishmanial infection, Adv. Exp. Med. Biol. 749 (2012), 201-213. doi:10.1007/978-1-4614-3381-1_14.

[10] A. Chaudhuri and A. Chattopadhyay, Transbilayer organization of membrane cholesterol at low concentrations: Implications in health and disease, Biochim. Biophys. Acta 1808 (2011), 19-25. doi:10.1016/j.bbamem.2010.10.013.

[11] J. Fantini and F.J. Barrnates, Sphingolipid/cholesterol regulation of neurotransmitter receptor conformation and function, Biochim. Biophys. Acta 1788 (2009), 2345-2361. doi:10.1016/j.bbamem.2009.08.016.

[12] J. Gatfield and J. Pieters, Essential role for cholesterol in entry of mycobacteria into macrophages, Science 288 (2000), 1647-1650. doi:10.1126/science.288.5471.1647.

[13] S. Gill, R. Chow and A.J. Brown, Sterol regulators of cholesterol homeostasis and beyond: The oxysterol hypothesis revisited and revised, Prog. Lipid Res. 47 (2008), 391-404. doi:10.1016/j.plipres.2008.04.002.

[14] G. Gimpl, Interaction of G protein coupled receptors and cholesterol, Chem. Phys. Lipids 199 (2016), 61-73. doi:10.1016/ j.chemphyslip.2016.04.006.

[15] S. Haldar and A. Chattopadhyay, Application of NBD-labeled lipids in membrane and cell biology, Springer Ser. Fluoresc. 13 (2013), 37-50. doi:10.1007/4243_2012_43.

[16] J.S. Harris, D.E. Epps, S.R. Davio and F.J. Kézdy, Evidence for transbilayer, tail-to-tail cholesterol dimers in dipalmitoylglycerophosphocholine liposomes, Biochemistry 34 (1995), 3851-3857. doi:10.1021/bi00011a043.

[17] M. Jafurulla and A. Chattopadhyay, Membrane lipids in the function of serotonin and adrenergic receptors, Curr. Med. Chem. 20 (2013), 47-55. doi:10.2174/0929867311302010006.

[18] M. Jafurulla, S. Tiwari and A. Chattopadhyay, Identification of cholesterol recognition amino acid consensus (CRAC) motif in G-protein coupled receptors, Biochem. Biophys. Res. Commun. 404 (2011), 569-573. doi:10.1016/j.bbrc.2010. 12.031.

[19] S. Keshavjee and P.E. Farmer, Tuberculosis, drug resistance, and the history of modern medicine, N. Engl. J. Med. 367 (2012), 931-936. doi:10.1056/NEJMra1205429. 
[20] G.A. Kumar, M. Jafurulla and A. Chattopadhyay, The membrane as the gatekeeper of infection: Cholesterol in hostpathogen interaction, Chem. Phys. Lipids 199 (2016), 179-185. doi:10.1016/j.chemphyslip.2016.02.007.

[21] G.A. Kumar, S. Roy, M. Jafurulla, C. Mandal and A. Chattopadhyay, Statin-induced chronic cholesterol depletion inhibits Leishmania donovani infection: Relevance of optimum host membrane cholesterol, Biochim. Biophys. Acta 1858 (2016), 2088-2096. doi:10.1016/j.bbamem.2016.06.010.

[22] D. Lingwood and K. Simons, Lipid rafts as a membrane-organizing principle, Science 327 (2010), 46-50. doi:10.1126/ science.1174621.

[23] L.M.S. Loura and M. Prieto, Dehydroergosterol structural organization in aqueous medium and in a model system of membranes, Biophys. J. 72 (1997), 2226-2236. doi:10.1016/S0006-3495(97)78866-0.

[24] M. Maccarrone, L. Bellincampi, G. Melino and A.F. Agrò, Cholesterol, but not its esters, triggers programmed cell death in human erythroleukemia K562 cells, Eur. J. Biochem. 253 (1998), 107-113. doi:10.1046/j.1432-1327.1998.2530107.x.

[25] D.H. Mauch, K. Nägler, S. Schumacher, C. Göritz, E.-C. Müller, A. Otto and F.W. Pfrieger, CNS synaptogenesis promoted by glia-derived cholesterol, Science 294 (2001), 1354-1357. doi:10.1126/science.294.5545.1354.

[26] O.G. Mouritsen, The liquid-ordered state comes of age, Biochim. Biophys. Acta 1798 (2010), 1286-1288. doi:10.1016/j. bbamem.2010.02.017.

[27] O.G. Mouritsen and M.J. Zuckermann, What's so special about cholesterol? Lipids 39 (2004), 1101-1113. doi:10.1007/ s11745-004-1336-x.

[28] S. Mukherjee and A. Chattopadhyay, Membrane organization at low cholesterol concentrations: A study using 7nitrobenz-2-oxa-1,3-diazol-4-yl-labeled cholesterol, Biochemistry 35 (1996), 1311-1322. doi:10.1021/bi951953q.

[29] S. Mukherjee and A. Chattopadhyay, Monitoring cholesterol organization in membranes at low concentrations utilizing the wavelength-selective fluorescence approach, Chem. Phys. Lipids 134 (2005), 79-84. doi:10.1016/j.chemphyslip.2004. 12.001 .

[30] S. Mukherjee and F.R. Maxfield, Membrane domains, Annu. Rev. Cell Dev. Biol. 20 (2004), 839-866. doi:10.1146/ annurev.cellbio.20.010403.095451.

[31] J. Oates and A. Watts, Uncovering the intimate relationship between lipids, cholesterol and GPCR activation, Curr. Opin. Struct. Biol. 21 (2011), 802-807. doi:10.1016/j.sbi.2011.09.007.

[32] S. Oddi, E. Dainese, F. Fezza, M. Lanuti, D. Barcaroli, V. De Laurenzi, D. Centonze and M. Maccarrone, Functional characterization of putative cholesterol binding sequence (CRAC) in human type-1 cannabinoid receptor, J. Neurochem. 116 (2011), 858-865. doi:10.1111/j.1471-4159.2010.07041.x.

[33] Y.D. Paila and A. Chattopadhyay, The function of G-protein coupled receptors and membrane cholesterol: Specific or general interaction? Glycoconj. J. 26 (2009), 711-720. doi:10.1007/s10719-008-9218-5.

[34] Y.D. Paila and A. Chattopadhyay, Membrane cholesterol in the function and organization of G-protein coupled receptors, Subcell. Biochem. 51 (2010), 439-466. doi:10.1007/978-90-481-8622-8_16.

[35] Y.D. Paila, M.R.V.S. Murty, M. Vairamani and A. Chattopadhyay, Signaling by the human serotonin ${ }_{1 \mathrm{~A}}$ receptor is impaired in cellular model of Smith-Lemli-Opitz syndrome, Biochim. Biophys. Acta 1778 (2008), 1508-1516. doi:10.1016/ j.bbamem.2008.03.002.

[36] Y.D. Paila, B. Saha and A. Chattopadhyay, Amphotericin B inhibits entry of Leishmania donovani into primary macrophages, Biochem. Biophys. Res. Commun. 399 (2010), 429-433. doi:10.1016/j.bbrc.2010.07.099.

[37] Y.D. Paila, S. Tiwari and A. Chattopadhyay, Are specific nonannular cholesterol binding sites present in G-protein coupled receptors? Biochim. Biophys. Acta 1788 (2009), 295-302. doi:10.1016/j.bbamem.2008.11.020.

[38] M. Perouansky, The Overton in Meyer-Overton: A biographical sketch commemorating the 150th anniversary of Charles Ernest Overton's birth, Br. J. Anaesth. 114 (2015), 537-541. doi:10.1093/bja/aev069.

[39] K.L. Pierce, R.T. Premont and R.J. Lefkowitz, Seven-transmembrane receptors, Nat. Rev. Mol. Cell Biol. 3 (2002), 639650. doi:10.1038/nrm908.

[40] F.D. Porter and G.E. Herman, Malformation syndromes caused by disorders of cholesterol synthesis, J. Lipid Res. 52 (2011), 6-34. doi:10.1194/jlr.R009548.

[41] J.A. Porter, K.E. Young and P.A. Beachy, Cholesterol modification of hedgehog signaling proteins in animal development, Science 274 (1996), 255-259. doi:10.1126/science.274.5285.255.

[42] A.V. Prabhu, W. Luu, D. Li, L.J. Sharpe and A.J. Brown, DHCR7: A vital enzyme switch between cholesterol and vitamin D production, Prog. Lipid Res. 64 (2016), 138-181. doi:10.1016/j.plipres.2016.09.003.

[43] X. Prasanna, D. Sengupta and A. Chattopadhyay, Cholesterol-dependent conformational plasticity of GPCR dimers, Sci. Rep. 6 (2016), 31858. doi:10.1038/srep31858.

[44] T.J. Pucadyil and A. Chattopadhyay, Role of cholesterol in the function and organization of G-protein coupled receptors, Prog. Lipid Res. 45 (2006), 295-333. doi:10.1016/j.plipres.2006.02.002.

[45] T.J. Pucadyil and A. Chattopadhyay, Cholesterol: A potential therapeutic target in Leishmania infection? Trends Parasitol. 23 (2007), 49-53. doi:10.1016/j.pt.2006.12.003.

[46] T.J. Pucadyil, S. Mukherjee and A. Chattopadhyay, Organization and dynamics of NBD-labeled lipids in membranes analyzed by fluorescence recovery after photobleaching, J. Phys. Chem. B 111 (2007), 1975-1983. doi:10.1021/jp066092h. 
[47] T.J. Pucadyil, P. Tewary, R. Madhubala and A. Chattopadhyay, Cholesterol is required for Leishmania donovani infection: Implications in leishmaniasis, Mol. Biochem. Parasitol. 133 (2004), 145-152. doi:10.1016/j.molbiopara.2003.10.002.

[48] N.E. Rodríguez, U. Gaur and M.E. Wilson, Role of caveolae in Leishmania chagasi phagocytosis and intracellular survival in macrophages, Cell. Microbiol. 8 (2006), 1106-1120. doi:10.1111/j.1462-5822.2006.00695.x.

[49] D.M. Rosenbaum, S.G.F. Rasmussen and B.K. Kobilka, The structure and function of G-protein-coupled receptors, Nature 459 (2009), 356-363. doi:10.1038/nature08144.

[50] R. Rukmini, S.S. Rawat, S.C. Biswas and A. Chattopadhyay, Cholesterol organization in membranes at low concentrations: Effects of curvature stress and membrane thickness, Biophys. J. 81 (2001), 2122-2134. doi:10.1016/S00063495(01)75860-2.

[51] D. Sengupta and A. Chattopadhyay, Identification of cholesterol binding sites in the serotonin 1 A receptor, J. Phys. Chem. B 116 (2012), 12991-12996. doi:10.1021/jp309888u.

[52] D. Sengupta and A. Chattopadhyay, Molecular dynamics simulations of GPCR-cholesterol interaction: An emerging paradigm, Biochim. Biophys. Acta $\mathbf{1 8 4 8}$ (2015), 1775-1782. doi:10.1016/j.bbamem.2015.03.018.

[53] E. Sevcsik and G.J. Schütz, With or without rafts? Alternative views on cell membranes, Bioessays 38 (2015), 129-139. doi:10.1002/bies.201500150.

[54] K. Simons and E. Ikonen, How cells handle cholesterol, Science 290 (2000), 1721-1726. doi:10.1126/science.290.5497. 1721.

[55] P. Tewary, K. Veena, P.J. Pucadyil, A. Chattopadhyay and R. Madhubala, The sterol-binding antibiotic nystatin inhibits entry of non-opsonized Leishmania donovani into macrophages, Biochem. Biophys. Res. Commun. 339 (2006), 661-666. doi:10.1016/j.bbrc.2005.11.062.

[56] D.E. Vance and H. Van den Bosch, Cholesterol in the year 2000, Biochim. Biophys. Acta 1529 (2000), 1-8.

[57] G. Viswanathan, M. Jafurulla, G.A. Kumar, T.R. Raghunand and A. Chattopadhyay, Dissecting the membrane cholesterol requirement for mycobacterial entry into host cells, Chem. Phys. Lipids 189 (2015), 19-27. doi:10.1016/j.chemphyslip. 2015.05.006. 